\title{
An efficient approach for processing skyline queries in incomplete multidimensional database
}

\begin{abstract}
In recent years, there has been great attention given to skyline queries that incorporate and provide more flexible query operators that return data items (skylines) which are not being dominated by other data items in all dimensions (attributes) of the database. Many variations in skyline techniques have been proposed in the literature. However, most of these techniques determine skylines by assuming that the values of all dimensions for every data item are available (complete). But this assumption is not always true particularly for large multidimensional database as some values may be missing (not applicable during the computation). In this paper, we proposed an efficient approach for processing skyline queries in incomplete database. The experimental results show that our proposed approach has significantly reduced the number of pairwise comparisons and the processing time in determining the skylines compared to the previous approaches.
\end{abstract}

Keyword: Skyline; Skyline queries; Preference queries; Incomplete database; Multidimensional database; Query processing 